preprint: THU-98/03 ; ULB-TH/99-05

\title{
How the Change in Horizon Area Drives Black Hole Evaporation
}

\author{
S. Massar \\ Service de Physique Théorique, Université Libre de Bruxelles, \\ CP 225, Bvd. du Triomphe, B1050 Brussels, Belgium \\ e-mail: smassar@ulb.ac.be

\begin{abstract}
R. Parentani
Laboratoire de Mathématiques et Physique Théorique, CNRS UPRES A 6083, Faculté des Sciences, Université de Tours, 37200 Tours, France.

e-mail: parenta@celfi.phys.univ-tours.fr
\end{abstract}

\begin{abstract}
We rephrase the derivation of black hole radiation so as to take into account, at the level of transition amplitudes, the change of the geometry induced by the emission process. This enlarged description reveals that the dynamical variables which govern the emission are the horizon area and its conjugate time variable. Their conjugation is established through the boundary term at the horizon which must be added to the canonical action of general relativity in order to obtain a well defined action principle when the area varies. These coordinates have already been used by Teitelboim and collaborators to compute the partition function of a black hole. We use them to show that the probability to emit a particle is given by $e^{-\Delta A / 4}$ where $\Delta A$ is the decrease in horizon area induced by the emission. This expression improves Hawking result which is governed by a temperature (given by the surface gravity) in that the specific heat of the black hole is no longer neglected. The present derivation of quantum black hole radiation is based on the same principles which are used to derive the first law of classical black hole thermodynamics. Moreover it also applies to quantum processes associated with cosmological or acceleration horizons. These two results indicate that not only black holes but all event horizons possess an entropy which governs processes according to quantum statistical thermodynamics.
\end{abstract}




\section{Introduction}

There are two possible approaches to the gravitational back reaction to Hawking radiation. The first is to develop a microscopic theory of quantum gravity and to use it to calculate the properties of black holes. This program has been partially realized in the context of super-string theory [1]. The second is to use Hawking's calculation[2] as a starting point to compute the gravitational corrections to black hole evaporation. Hopefully these two approaches should meet in some middle ground.

In the second approach, the back reaction has been addressed along two complementary lines. The first is the "semi-classical" theory wherein one first computes the mean (quantum average) energy-momentum tensor of the quantized fields propagating on the background geometry and then solves the Einstein equations driven by this mean value. The solution describes an evaporating geometry characterized by the shrinking of the horizon area [3, 4, 5. In this treatment, the metric remains classical and unaffected by the quantum fluctuations of the energy-momentum tensor.

The second line of attack is to take into account the dynamics of gravity at the level of transition amplitudes before performing the quantum average over the configurations of the radiation field. Since Hawking radiation is derived from quantum field theory, the most natural procedure would be to use Feynman rules. However, the ill-defined ultraviolet behavior of quantum gravity has so far prevented this approach from being successfully followed. Thus, one has to resort to less ambitious schemes.

Some interesting insights have been obtained by taking the gravitational back reaction into account at the classical level, before computing transition amplitudes. To this end, the matter action $S_{\text {matter }}$ in a given geometry is replaced by the action of matter plus gravity $S_{\text {matter+gravity }}$ A concrete model has been developed by Keski-Vakkuri, Kraus and Wilczek (KKW) [6, 7, 8]. It describes the propagation of a massless spherically symmetric self-gravitating shell in a black hole geometry. Having computed the new action, they postulate that the wave function of a shell is given by the WKB form $e^{i S_{m+g}}$. Using these waves, they derive the black hole emission amplitudes of uninteracting (dilute gas approximation) shells. A similar approach has been used in a Euclidean framework in [9] following the techniques developed in [10]. In this case, the probability for the black hole to emit a particle is expressed in terms of the action $S_{m+g}$ of a self gravitating instanton.

The striking result of these works is that the probability for a black hole of mass $M$ to emit a particle of energy $\lambda$ is given by

$$
P_{M \rightarrow M-\lambda}=N(\lambda, M) e^{-\Delta A(\lambda, M) / 4}
$$

where $\Delta A(\lambda, M)=A(M)-A(M-\lambda)$ is the decrease of the area of the black hole horizon. $N$ is a phase space (also called grey body) factor which cannot be calculated in these approximation schemes.

Eq. (11) replaces Hawking's result $P_{M \rightarrow M-\lambda}=N e^{-2 \pi \lambda / \kappa}$ which is characterized by a temperature $T_{H}=\kappa / 2 \pi$ defined by the surface gravity $\kappa$. To first order in $\lambda$, the first law 11] of classical black hole thermodynamics $d E=\frac{\kappa}{8 \pi} d A$ guarantees that the two expressions for $P_{M \rightarrow M-\lambda}$ coincide. This suggests that Hawking radiation and the first 
law both stem from the same principle. This is far from obvious since the first law relates neighboring classical solutions of general relativity whereas black hole radiance is derived from QFT in a given geometry.

The aim of the present work is to reveal their common origin and to establish the universal validity of eq. (11). We shall show that both follow from the use of the complete action $S_{m+g}$ in place of the matter action in a given geometry. The reason is that the emission of a matter quantum can now be viewed as the transition between two neighboring black hole states. In this transition, the energy is transfered from the hole to the radiation, a feature absent in the derivation of black hole radiance based on Bogoliubov coefficients evaluated in a given geometry. Moreover, the relevant dynamical quantity governing this transition is the difference of actions: $S_{m+g}($ final $)-S_{m+g}($ initial $)$. Then, as in deriving the first law [12, this difference can be reexpressed as a difference of boundary terms at the horizon. From this, it is easy to show that the dynamical quantity which governs emission rates is equal to the change in horizon area divided by 4 .

To demonstrate this result, we make use of the appropriate coordinate system to describe processes inducing changes in area. These are the boost parameter $\Theta$ and its conjugate variable, the area of the horizon $A / 8 \pi$. These variables where used by Teitelboim and collaborators [12, 13, 14, 15, 16] to compute the partition function of the black hole à la Gibbons-Hawking [17] starting from the appropriate classical action. In this case, they showed that one must add to the canonical action $(p d q-H d t)$ a boundary term equal to $\Theta A / 8 \pi$. In our context, it is through this boundary term that we shall obtain eq. (臬) and establish the relationship with the first law.

We have organized this paper as follows. After a brief review of Hawking's derivation formulated in a fixed geometry, we introduce the reader to the variables $\Theta$ and $A$ and to their role in boundary terms at the horizon. In particular we emphasize that the particular form of the boundary term is dictated by the physical process considered. For the emission of particles by a black hole, the appropriate action is the canonical action $S_{m+g}$ supplemented by $\Theta A / 8 \pi$.

This action is used in section 1 to compute the transition rates of a detector at a fixed radius of the black hole. The usefulness of introducing a detector is that the transition amplitudes are expressed in terms of the overlap of the initial and final states of the detector+black hole complex. Both are stationary eigenvectors of $i \partial_{\Theta}$ with eigenvalue equal to the area of the black hole. In this setting the ADM mass is fixed and therefore the time at infinity cannot be used to parameterize the evolution 18]. Instead one must use $\Theta$ time. It is then straightforward to show that the ratio of transition rates of the detector is given by $e^{-\Delta A / 4}$.

Even though the introduction of a detector is useful, it is not intrinsic to black hole radiation. This is why in section 5 we reconsider the KKW model which only makes appeal to the action of the particles emitted by the black hole. By using $\Theta$ and $A$ we shall recover KKW's result in very simple terms and make contact with the former derivation. We shall also show that eq. (四) follows directly from the universal form of out-going trajectories in the near horizon geometry and the specification that the field configurations be in (Unruh) vacuum. Thus eq. (11) applies to all emission processes in the presence of horizons, including charged and rotating black hole, cosmological and 
acceleration horizons (in the latter case the horizon area is infinite, but differences are finite and well-defined, see [10, 9, 19]).

The universal validity of this derivation is the main result of this paper. It proves that the area of all event horizons determines the gravitational statistical entropy available [20] to these quantum processes. In this we give statistical foundations 21] to the relation between horizon thermodynamics and Einstein's equations exhibited by Jacobson[22].

\section{Hawking Radiation}

In this section we fix the notations and review two standard derivations of black hole radiation. In the first derivation, following Unruh[23], we introduce a two level atom coupled to the radiation field and whose position is fixed. Then, using Einstein's argument, one determines the distribution of massless quanta from its transition rates. In this way one only uses basic quantum mechanical rules. The second approach is more intrinsic and closer to Hawking's derivation[2]. Black hole radiation is established through the Bogoljubov transformation relating in-modes which determine the state of the radiation field before the collapse (which shall be taken for simplicity to be in-vacuum) and out-modes which define the particles emerging from the hole and found at infinity.

The metric of a Schwarzschild black hole is

$$
d s^{2}=-\left(1-\frac{2 M}{r}\right) d t^{2}+\left(1-\frac{2 M}{r}\right)^{-1} d r^{2}+r^{2} d \Omega^{2}
$$

We introduce the light like coordinates $u$ and $v$ :

$$
v, u=t \pm r^{*} \quad, \quad r^{*}=r+2 M \ln |r-2 M|
$$

and the Kruskal coordinates $U_{K}$ and $V_{K}$ :

$$
U_{K}=-\frac{1}{\kappa} e^{-\kappa u} \quad, \quad V_{K}=\frac{1}{\kappa} e^{\kappa v}
$$

where $\kappa=1 / 4 M$ is the surface gravity.

For a black hole formed by the collapse of a spherically symmetric star, the outgoing modes, solutions of the Dalembertian equation have the following form near the horizon, i.e. for $r-2 M \ll 2 M$ :

$$
\phi_{\omega l m}=\frac{e^{-i \omega U_{K}}}{\sqrt{4 \pi \omega}} \frac{Y_{l m}(\Omega)}{r} .
$$

Further away from the horizon this expression is no longer exact because of the potential barrier which surrounds the black hole. For simplicity, throughout the article, we shall neglect the transmission coefficients (also called grey body factors) induced by this barrier since they cancel out from the ratio of the transition rates which determines the equilibrium distribution around an eternal black hole, see eq. (10) below. This cancellation also applies to charged and rotating black holes when one takes into account the "work term" exerted by the electric potential or the angular momentum of the hole, see 24, 25. 
The modes eq. (5) are associated with the out-going particles as seen by infalling observers. Indeed, for $r-2 M \ll 2 M$, the proper time lapse of infalling observers is proportional to $\Delta U_{K}$. This is simply seen by re-expressing the Schwarzschild metric in $U_{K}, V_{K}$ coordinates: $d s^{2} \simeq-d U_{K} d V_{K}+r^{2} d^{2} \Omega$. Hence near the horizon, for infalling observers, the modes eq. (5) have positive frequency.

Using these modes, the field operator can be decomposed as:

$$
\Phi=\sum_{\omega, l, m} a_{\omega l m} \phi_{\omega l m}+\text { h.c. }+ \text { ingoing modes . }
$$

By definition, the in-vacuum state, denoted $\left|0_{U}\right\rangle$, is annihilated by the $a_{\omega l m}$ operators. In the literature it is often called the Unruh vacuum. The fact that it is annihilated by all $a_{\omega l m}$ guarantees that infalling observers experience vacuum conditions as they cross the future horizon.

Consider now a particle detector at fixed radius $R$ of the black hole and angular position $\Omega$. It has two levels $|e\rangle$ and $|g\rangle$ of energy $E_{e}$ and $E_{g}$ with $\Delta E=E_{e}-E_{g}>0$. For simplicity we shall take $R$ to be very large $(R \gg 2 M)$. Then $t$ is the proper time of the detector and $\Delta E$ is the energy gap as measured from $r=\infty$. For smaller $r$, one should take into account the gravitational red shift.

In the interaction representation, the coupling of the detector to the field $\Phi$ is given by

$$
H_{\text {int }}=\gamma \Phi(t, R, \Omega)\left(e^{+i \Delta E t}|e\rangle\langle g|+\text { h.c. }\right)
$$

where $\gamma$ is the coupling constant.

When the detector is initially in its ground state, the state of the system (detector plus radiation field $\Phi$ ) is $\left|0_{U}\right\rangle|g\rangle$. In the interacting picture, at late times and to first order in $\gamma$, this state becomes:

$$
\begin{aligned}
\left|\psi_{g}\right\rangle & =\left|0_{U}\right\rangle|g\rangle-i \gamma \int d t e^{i \Delta E t} \Phi(R, \Omega, t)\left|0_{U}\right\rangle|e\rangle \\
& =\left|0_{U}\right\rangle|g\rangle-i \gamma \sum_{\omega l m} \int d t e^{i \Delta E t} e^{-i \omega C e^{-\kappa t}} \frac{Y_{l m}^{*}(\Omega)}{R \sqrt{4 \pi \omega}} a_{\omega l m}^{\dagger}\left|0_{U}\right\rangle|e\rangle \\
& =\left|0_{U}\right\rangle|g\rangle+\sum_{\omega l m} B_{g \rightarrow e, \omega, l, m} a_{\omega l m}^{\dagger}\left|0_{U}\right\rangle|e\rangle
\end{aligned}
$$

where $C=(1 / \kappa) \exp \left(\kappa R+\frac{1}{2} \ln (R-2 M)\right)$.

Similarly if the detector was initially in its excited state, the final state would have been

$$
\begin{aligned}
\left|\psi_{e}\right\rangle & =\left|0_{U}\right\rangle|e\rangle-i \gamma \sum_{\omega l m} \int d t e^{-i \Delta E t} e^{-i \omega C e^{-\kappa t}} \frac{Y_{l m}^{*}(\Omega)}{R \sqrt{4 \pi \omega}} a_{\omega l m}^{\dagger}\left|0_{U}\right\rangle|g\rangle \\
& =\left|0_{U}\right\rangle|e\rangle+\sum_{\omega l m} B_{e \rightarrow g, \omega, l, m} a_{\omega l m}^{\dagger}\left|0_{U}\right\rangle|g\rangle
\end{aligned}
$$

Since we are interested in determining the population of quanta seen by the detector, we only need to compute the ratio of the transition rates. By replacing $t$ by $t+i \pi / \kappa$ in the 
integral governing the transition amplitude $B_{e \rightarrow g, \omega, l, m}$, one obtains $B_{g \rightarrow e}=B_{e \rightarrow g}^{*} e^{-\pi \Delta E / \kappa}$ for all $l, m, \omega$, see [26, 27]. Thus the ratio of the transition probabilities is:

$$
\frac{\left|B_{g \rightarrow e, \omega, l, m}\right|^{2}}{\left|B_{e \rightarrow g, \omega, l, m}\right|^{2}}=e^{-\frac{2 \pi}{\kappa} \Delta E} \text {. }
$$

This corresponds to the rates in a thermal bath at temperature $\kappa / 2 \pi=1 / 8 \pi M$. Then, using Einstein's argument, one obtains that the quanta of the radiation field are distributed according to the Planck distribution.

This derivation is equivalent to calculating the Bogoljubov transformation between Unruh modes, eq. (5), and the out-modes defined below in eq. (11). Indeed the transition amplitudes $B_{g \rightarrow e, \omega, l=0}$ and $B_{e \rightarrow g, \omega, l=0}$ are proportional to the Bogoliubov coefficients $\alpha_{\omega \lambda}$ and $\beta_{\omega \lambda}$ when $\Delta E=\lambda$, see [23, 26]. This relationship provides a physical interpretation of Bogoljubov coefficients as transition amplitudes. A concept that we shall rediscuss below.

We now turn to the second derivation in which the spectrum of emitted particles is determined only in terms of solutions of the Dalembertian with a given frequency $i \partial_{t}=\lambda$. There are two positive norm modes for each value of $\lambda$ which identically vanish either inside or outside the horizon:

$$
\begin{array}{ll}
\varphi_{\lambda,+}=\frac{e^{-i \lambda u}}{\sqrt{4 \pi \lambda}} \frac{1}{r} \theta(r-2 M), & \lambda>0, \\
\varphi_{\lambda,-}=\frac{e^{+i \lambda u}}{\sqrt{4 \pi \lambda}} \frac{1}{r} \theta(2 M-r), \quad \lambda>0 .
\end{array}
$$

Once more we have neglected the potential barrier outside the black hole and for simplicity we have considered only s-waves $(l=0)$.

The modes $\varphi_{+}$define the out-quanta, i.e. those used by a static observer around the black hole to describe the presence or absence of out-going particles. We can again decompose the field operator into $\varphi_{ \pm}$modes:

$$
\Phi=\sum_{\lambda, \pm} a_{\text {out }, \lambda \pm} \varphi_{\lambda, \pm}+\text { h.c. }+ \text { ingoing modes . }
$$

By definition the $a_{\text {out }, \lambda \pm}$ annihilate the out vacuum, $\left|0_{\text {out }}\right\rangle$.

It is now appropriate to introduce a third set of modes $\phi_{\lambda, \pm}$ which possess the following properties. They are eigenmodes of $i \partial_{t}=\lambda$ and are composed only of positive frequency modes, eq. (5), which define Unruh vacuum. The simplest way to implement this last condition is to express the out-modes in Kruskal coordinates: $\varphi_{\lambda \pm} \simeq$ $\left(\mp U_{K}\right)^{ \pm i \lambda / \kappa} \theta\left(\mp U_{K}\right)$. Since $\Delta U_{K}$ is proportional to the proper time of an infalling observer, $\phi_{\lambda}$ must be the linear combination of $\varphi_{\lambda \pm}$ which is analytic and bounded in the lower half of the complex $U_{K}$ plane. Upon requiring also that the $\phi_{\lambda \pm}$ have unit norm, one obtains

$$
\begin{aligned}
& \phi_{\lambda+}=\frac{1}{\sqrt{1-e^{-2 \pi \lambda / \kappa}}}\left(\varphi_{\lambda+}+e^{-\pi \lambda / \kappa} \varphi_{\lambda-}^{*}\right), \quad \lambda>0 \\
& \phi_{\lambda-}=\frac{1}{\sqrt{1-e^{-2 \pi \lambda / \kappa}}}\left(\varphi_{\lambda-}+e^{-\pi \lambda / \kappa} \varphi_{\lambda+}^{*}\right), \quad \lambda>0 .
\end{aligned}
$$


One can verify by evaluating the overlap of $\phi_{\lambda}$ and $\phi_{\omega}$ that the $\phi_{\lambda}$ are linear combinations of the $\phi_{\omega}$ with no $\phi_{\omega}^{*}$ component, see e.g. [27]. One can then decompose the field operator in terms of these new modes:

$$
\Phi=\sum_{\lambda, \pm} a_{\lambda \pm} \phi_{\lambda, \pm}+\text { h.c. }+ \text { ingoing modes } .
$$

Then, Unruh vacuum is annihilated by the $a_{\lambda \pm}$ operators.

The weights in eq. (13) define the Bogoljubov coefficients $\alpha_{\lambda}$ and $\beta_{\lambda}$. Their ratio satisfies

$$
\frac{\left|\beta_{\lambda}\right|^{2}}{\left|\alpha_{\lambda}\right|^{2}}=e^{-2 \pi \lambda / \kappa} \text {. }
$$

Since $\left|\beta_{\lambda}\right|^{2}$ determines the mean number of out quanta of energy $\lambda$ in Unruh vacuum, eq. (15) implies that Unruh vacuum is a thermal distribution of out-particles at temperature $\kappa / 2 \pi$, in agreement with eq. (10).

We will find it convenient below to use the same argument, but rephrased in coordinate systems which are regular on the future horizon and which lead to a static metric. This second condition implies that the time parameter is proportional to $t$ at fixed $r$. An example is given by the Eddington-Finkelstein coordinates $v, r, \Omega$ in which the metric has the form:

$$
d s^{2}=-(1-2 M / r) d v^{2}+2 d v d r+r^{2} d \Omega^{2}
$$

Near the horizon the metric takes the simple form $d s^{2} \simeq 2 d v d r+r^{2} d \Omega^{2}$ which shows that $v, r$ are inertial coordinates. Moreover since $-d r$ is proportional to $d U_{K}$, the momentum $p_{r}$ plays the role of the frequency $\omega$ of eq. (5) and one can translate the analytical condition implementing Unruh vacuum in terms of $r$ : to obtain a $\phi$ mode, one analytically continues $\varphi_{+}$in the upper half complex $r$ plane to define its value for $r<2 M$. As shown in [28], one immediately obtains eq. (13). In Section 5, it is through the analytical behavior in $r$ of the modified modes that we shall determine the corrections to eq. (15).

In conclusion of this Section, we wish to emphasize the following point. In the transitions described in eq. (8), there is a transfer of energy from the radiation field to the detector but the black hole mass stays constant. Similarly, upon computing the Bogoliubov coefficients in eq. (13), the geometry is unaffected. In these descriptions of black hole radiation, there is no transfer of energy from the hole into radiation. The notion of black hole evaporation only arises when the mean energy momentum tensor of the radiated quanta is put on the r.h.s of Einstein's equations wherein it drives the shrinking of the horizon area[2]-[5].

This artificial two step procedure results from the original sin: to have decided to work in a given geometry. As we shall see in Sections 4 and 5, upon working with a recoiling geometry, energy conservation will be taken into account at the level of amplitudes, as in the Compton effect. Then, the black hole will act as a conventional reservoir of energy: when delivering heat to the external world it loses the corresponding energy. 


\section{Boundary terms in the Einstein-Hilbert Action}

In this section, following [12, 13, 14], we introduce coordinates which have an intrinsic geometric interpretation near the horizon. These coordinates are the hyperbolic angle $\Theta$, the transverse coordinates along the horizon $x_{\perp}^{i}$ and the radial proper distance from the horizon $\rho$. In terms of these coordinates, the metric near the horizon takes the universal form

$$
d s^{2} \simeq-\rho^{2} d \Theta^{2}+d \rho^{2}+\gamma_{i j} d x_{\perp}^{i} d x_{\perp}^{j}
$$

For simplicity we have written the metric for a spherically symmetric horizon, for the general case we refer to [14]. The area of the horizon is $A=\left.\int d x_{\perp}^{2} \sqrt{\gamma}\right|_{\rho=0}$. In the case of a Schwarzschild on-shell solution, $\Theta=\kappa t_{\infty}$ and $\rho \simeq \sqrt{8 M(r-2 M)}$ where $t_{\infty}$ is the proper time at spatial infinity.

The universal form of the metric in these coordinates implies that if we use them to describe a process near a particular horizon, the description of the same process in the vicinity of any other horizon will be identical. The developments presented in sections 4 and 5 for a Schwarzschild black hole thus also apply to charged and rotating holes 15 and to cosmological and acceleration horizons. Furthermore because these coordinates lead to such a simple form for the metric, physical process occurring near the horizon will be most simply described in these coordinates. In this section, this will be illustrated by considering the boundary terms at the horizon that arise in the Hamiltonian formulation of general relativity. In the next sections we shall see that these coordinates are also well adapted to describe particle production near event horizons.

We start the analysis of the boundary terms in the canonical action for matter and gravity

$$
I_{c a n}=\int_{0}^{t} d t^{\prime}\left\{\pi^{a b} \dot{g}_{a b}+p \dot{q}-N H-N^{i} H_{i}\right\}
$$

where $g_{a b}$ is the spatial metric, $\pi^{a b}$ its conjugate momentum, $q$ and $p$ the coordinates and momentum of matter, $N$ and $N^{i}$ the lapse and shift, and $H$ and $H_{i}$ the energy and momentum constraints. In what follows, we focus on metrics $g_{a b}$ which have two spatial boundaries. We suppose that at one boundary the metric is asymptotically flat, that is $g_{a b}$ and $\pi^{a b}$ tend to their value in flat space as the proper distance $\rho \simeq r$ tends to infinity, see [29] for the precise behavior one imposes. We also suppose that at the other boundary the metric can be put in the form eq. (17), see [14 for the precise conditions imposed at this boundary. Thus we are considering the class of metrics which, on shell and in vacuum, will correspond to one of the asymptotically flat quadrants of an eternal black hole.

Because these metrics have spatial boundaries, it may be necessary to add boundary terms to $I_{\text {can }}$ in order for it to be stationary on the solutions of the equations of motion. We now review these features, starting with the boundary at infinity. Details of the calculations will not be presented. They can be found in many papers, see for instance [29] [30] [16]. Let us first emphasize that the following considerations are superfluous for classical physics, i.e. for the determination of the solutions of the equations of motion. However, when considering quantum kernels, transition amplitudes or parti- 
tion functions, they cannot be ignored since the WKB value of the quantum phase is determined by the action.

To identify the boundary terms, following [13], we compute the total variation of the action given in eq. (18). By total variation we mean that we are considering the linear change of $I_{c a n}$ due to an arbitrary change of all its variables. We note that this variation can be performed around any configuration, i.e., on-shell or off-shell. We nevertheless impose that the space time is asymptotically flat. In this case, the first order change of $I_{\text {can }}$ contains three types of terms. First we have the contribution of the bulk, the 4-geometry interpolating the initial to the final 3-geometry. As usual, this contribution vanishes when the reference configuration is on-shell. Secondly, one has the contributions due to the change of the initial and final configuration. These determine (as usual) the initial and final momenta of gravity. Finally, one has an additional contribution arising from spatial infinity. On shell, it is is equal to

$$
\delta I_{\text {can }}^{r=\infty}=t_{\infty} \delta M_{A D M}
$$

where $t_{\infty}$ is the (coordinate invariant) proper time at spatial infinity. It is related to the lapse function $N(r)$ by $t_{\infty}=\int_{0}^{t} d t^{\prime} N\left(t^{\prime}, r=\infty\right) . \delta M_{A D M}$ is the change in mass at infinity which is defined by $\delta g_{r r}$ for large $r$. Eq. (19) shows that $I_{c a n}$ is extremal on shell, i.e. its variation reduces on shell to $\left.\pi^{a b} \delta g_{a b}\right|_{\text {initial }} ^{\text {final }}$, only if $\delta M_{A D M}=0$, i.e. only if one varies among the sub-class of metrics for which $M_{A D M}$ is kept fixed.

If one wishes to consider the Legendre conjugate ensemble in which the asymptotic proper time is fixed, one must work with the action $S^{\prime}=I_{c a n}-t_{\infty} M_{A D M}$ Indeed, the variation of this new action yields the following asymptotic contribution

$$
\delta S_{r=\infty}^{\prime}=-\delta t_{\infty} M_{A D M}
$$

This term vanishes when one works among the sub-class of metrics for which $t_{\infty}$ is kept fixed but $\delta M_{A D M}$ is arbitrary.

We now turn to the boundary term at the horizon. The analysis proceeds in parallel with the preceding one. The form of the boundary term is dictated by the fact that one requires that, near the horizon, the lapse and shift vanish (or more precisely that at $\rho=0$, the momentum $\pi_{\rho}^{\rho}$ and the derivative of the area of the surfaces of constant $\rho, \partial_{\rho} A$, vanish, see [14]) and hence the metric can be put in the form eq. (17). Upon varying eq. (18) one finds a boundary term at the horizon

$$
\delta I_{\text {can }}^{\text {inner boundary }}=-\Theta \delta A / 8 \pi
$$

Here $\delta A$ is the change in horizon area and $\Theta$ is the hyperbolic angle defined in eq. (17). It is equal to the limit $r \rightarrow r_{\text {horizon }}$ of $N^{2}(r) t / 2\left(r-r_{\text {hor }}\right)$. For simplicity of writing, we have again considered only spherically symmetric 3 -geometries. For the general case, see [14. Notice that $\Theta$ is a coordinate invariant quantity, exactly like $t_{\infty}$ in the asymptotic contribution. Moreover, as pointed out in [12], $A / 8 \pi$ and $\Theta$ are conjugate variables exactly like $M_{A D M}$ and $t_{\infty}$. Eq. (21) shows that $I_{c a n}$ is extremal on-shell for the subclass of metrics which have fixed horizon area. 
When one wishes to work with a fixed opening angle $\Theta$, one must work with the action $S=I_{c a n}+\Theta A / 8 \pi$ since its variation yields

$$
\delta S^{\text {inner boundary }}=A \delta \Theta / 8 \pi .
$$

$S$ is thus extremal on-shell for the class of metrics which have fixed $\Theta$. This action must be used when working in the Euclidean continuation of the black hole 16. Indeed regularity of the Euclidean manifold at the horizon imposes a fixed Euclidean angle given by $\Theta_{E}=2 \pi$. The surface term in the action then contributes a term $A / 4$ to the partition function which is interpreted as the entropy of the black hole. One of the main advantage of introducing this boundary term is to clarify the derivation of this partition function which was first considered by Gibbons and Hawking 17].

In brief, upon considering dynamical processes occurring around a black hole, there are a priori 4 actions which can be considered according to which quantities are fixed in the variational principle (or in the path integral) and therefore according to the surface terms. Two however are rather unphysical. Indeed fixing both the ADM mass and the horizon area is too constraining as can be seen by considering the vacuum spherically symmetric solutions for which fixing the ADM mass determines the horizon area (in a path integral, the kernel would vanish). For non empty geometries, this double specification would impose an unusual non-local constraint on the matter energy repartition. Similarly fixing both the time at infinity and the hyperbolic angle $\Theta$ is also too constraining. Thus one is left with two possibilities: fixing $\Theta$ and $M_{A D M}$ or fixing $t_{\infty}$ and $A$. A more mathematical reason why these are the only two possibilities (in the absence of matter) is that the constraints $H=0$ and $H_{i}=0$ viewed as differential equations need boundary conditions in order to yield a unique solution and fixing the ADM mass or the horizon area but not both provides the required boundary data 14.

The choice among the two remaining possibilities is dictated by physical considerations. If $M_{A D M}$ is not fixed but $A$ is, this means that one is considering configurations in which the ADM mass is determined by the repartition of matter surrounding the black hole while leaving the black hole unchanged. On the other hand, if one fixes $M_{A D M}$ while letting $A$ to be determined, one is exploring configurations in which energy can be redistributed between the black hole and the surrounding matter, but with no change of the ADM mass 凹.

Clearly black hole evaporation requires the second situation if one wants to analyze what happens at finite $r$. Therefore in the next section, we shall fix the mass at infinity and follow the evolution in terms of $\Theta$ and $A$. The relevant action in this case is

$$
S=\int_{0}^{t} d t^{\prime}\left\{\pi^{a b} \dot{g}_{a b}+p \dot{q}-N H-N^{i} H_{i}\right\}+\Theta(t) A / 8 \pi
$$

${ }^{1}$ To be complete, we should perhaps point out the following difficulty. The horizon is the $2 \mathrm{D}$ boundary common to all 3 -surfaces including the initial and the final ones. Hence, its area cannot vary in time. Therefore in order to allow $A$ to vary, one must consider a kind of regularized version in which the boundary of the 3 -surfaces is arbitrarily close to the horizon, but does not coincide with it. To our knowledge, the precise procedure has yet not been completely worked out. We mention here the recent works 31, 32, 33 in which generalized definitions of horizon have been proposed, mainly to allow for evolution. However, in what follows, we shall not need an action governing continuous changes in area. Only states corresponding to constant areas will be used. 
In section 5 we shall also consider the region behind the horizon. Thus the formalism developed here will not be directly applicable. Nevertheless, it will still be convenient to keep the ADM mass fixed and to describe the process in terms of $\Theta$ and $A$.

\section{Probability for detector transitions in terms of horizon area change}

In this section and the following one we calculate the transition rates governing black hole radiation when the matter action in a fixed geometry is replaced by the sum of matter and Einstein-Hilbert actions. In this new description, the change of the geometry induced by the transition process is taken into account through the extremisation of the total action. We start with the description of black hole radiation based on the transitions of a static detector.

Our aim is to show that it is the change in area associated with the quantum jump of the detector which determines the transition amplitudes. The detector is assumed to be at $r=R$ in one of the asymptotically flat quadrants of an eternal black hole. We believe however that our analysis also applies to black holes formed by collapse and that the mathematical analysis in the two cases should be identical, see however the last footnote. As in Section 2, we compute the transition amplitudes in perturbation theory to first order in the coupling $\gamma$ of the detector to the field, see eq. (7). As before they are given by the overlap of the three free waves. Here these are the radiation wave function and the two stationary states of the detector + black hole complex. These two stationary states have two interesting properties. Firstly, their eigenvalue is the black hole area rather than the detector's energy. Secondly, since the ADM mass is fixed, each eigenvalue is entangled to the corresponding detector state. Thus, in the new description, the transitions of the detector lead to quantum jumps from one horizon area to the other without smooth (classical) interpolation from one stationary geometry to the other.

We first compute the $\Theta$-time dependence of the wave functions associated with the two states of the detector when their energy is taken into account in the definition of the background geometry. This amounts to evaluate twice the on-shell action, eq. (23). Since the detector is at $r=$ const, both classical geometries are static. Thus the $p \dot{q}$ and

$\pi^{a b} \dot{g}_{a b}$ terms in the action vanish. Moreover, on-shell, the constraints also vanish. Hence, at fixed $M_{A D M}$, the only term contributing to $S$ is the surface term at the horizon. This term is equal to $\Theta A_{g} / 8 \pi$ or $\Theta A_{e} / 8 \pi$ where $A_{g}\left(A_{e}\right)$ is the horizon area when the detector is in the ground (excited) state. Thus the time dependence of the free (i.e. $\gamma=0$ ) wave functions are

$$
\begin{aligned}
& \Psi_{B H+g}(\Theta)=e^{i \Theta A_{g} / 8 \pi} \Psi_{B H+g}(0) \\
& \Psi_{B H+e}(\Theta)=e^{i \Theta A_{e} / 8 \pi} \Psi_{B H+e}(0)
\end{aligned}
$$

This is certainly correct in a WKB approximation. Moreover, since in the absence of interactions with the radiation field one deals with stationary area eigenstates, the 
exponential form is exact. Of course, in an exact quantum treatment, the eigen-areas may receive quantum corrections, but this will not affect the exponential behavior.

We now determine the expression for the outgoing modes which replaces eq. (5). In this section, we shall not take into account the gravitational deformation induced by these modes. We shall also neglect the effects that the fluctuations of the geometry [36, 37] might have. The validity of both hypothesis will be discussed after we have presented the mathematical outcome. In the absence of back-reaction and metric fluctuations, the modes must be such that they correspond to excitations of the vacuum state defined near the horizon. From the definition of $\Theta$, its relationship to the inertial light like coordinates $U_{K}, V_{K}$ (such that $d s^{2}=-d U_{K} d V_{K}+r^{2} d^{2} \Omega$ near the horizon) is of the form $d U_{K}=C e^{-\Theta} d \Theta$ at fixed $r$. This universal relation exhibits the exponential Doppler shift which is the hallmark of horizons (except for extremal black holes). Therefore, we make the hypothesis that the new expression is

$$
\phi_{\omega}(\Theta, R)=D e^{i \omega U_{K}}=D e^{i C \omega e^{-\Theta}}
$$

in place of $e^{i C \omega e^{-\kappa t}}$, see eqs. (8, 9). $D$ is a constant which plays no role.

We now assume that, to first order in $\gamma$ and as in eqs. (8, 9), the transition amplitudes are given by the "time" integral of the product of the three waves. Up to the same overall constant (see the discussion after eq. (5)), they then are given by

$$
\begin{aligned}
\mathcal{B}_{g \rightarrow e+\omega} & =\int d \Theta \Psi_{B H+g} \Psi_{B H+e}^{*} \phi_{\omega}^{*}, \\
\mathcal{B}_{e \rightarrow g+\omega} & =\int d \Theta \Psi_{B H+g}^{*} \Psi_{B H+e} \phi_{\omega}^{*} .
\end{aligned}
$$

Using eq. (24) and eq. (25) and by replacing $\Theta$ by $\Theta+i \pi$ in either amplitude, one obtains

$$
\frac{\left|\mathcal{B}_{g \rightarrow e+\omega}\right|^{2}}{\left|\mathcal{B}_{e \rightarrow g+\omega}\right|^{2}}=e^{\left(A_{e}-A_{g}\right) / 4}
$$

This establishes that $\left(A_{e}-A_{g}\right) / 4$, the difference of the horizon areas if the detector is excited or not, governs the equilibrium distribution of the detector's states. The new distribution clearly corresponds to a micro-canonical distribution since we are considering exchanges of energy between the black hole and the detector with no ADM mass change at spatial infinity. This confirms the interpretation of $A / 4$ as the statistical entropy of the black hole since $e^{A / 4}$ enters in eq. (27) as the quantum degeneracy of the initial and final black hole states. In this we confirm what has been found in [10, 19, 8].

Eq. (27) replaces the canonical expression of eq. (10) which is governed by the energy change $E_{e}-E_{g}$ and by Hawking temperature $\kappa / 2 \pi$. To first order in $E_{e}-E_{g}$, energy conservation and the (static version 11] of the) first law (i.e. $d E_{\text {detect. }}=-d M_{\text {hole }}=$ $-\kappa d A / 8 \pi)$ guarantee that the new expression gives back Hawking's result eq. (10). The correction to this first order approximation is governed by the specific heat of the black hole. Thus it is completely negligible for large black holes. Therefore the main changes from eq. (10) to eq. (27) are conceptual. First, energy conservation is now built in through the use of the extremised total action. Secondly, the thermalization of the detector no longer reveals that a thermal flux of photons is emitted by the hole but 
more fundamentally that the detector is in contact with a reservoir whose entropy is $A / 4$. Thirdly, the geometry jumps from one stationary situation to another one without smooth (classical) interpolation between them.

\section{Discussion}

In order to reveal the origin of these qualitative changes and to justify the hypothesis we made, it is appropriate to rewrite the transition amplitudes in terms of the complete system: black hole, detector and radiation field.

To quantize the whole system requires to consider the Wheeler-DeWitt constraints

$$
H_{\mu}^{B H+\text { detect. }+\Phi}|\Psi\rangle=0
$$

When $t$, the lapse of proper time at spatial flat infinity is fixed, the Wheeler-DeWitt equation is supplemented [12] by the following equation:

$$
i \partial_{t}|\Psi\rangle=\hat{M}_{A D M}|\Psi\rangle
$$

Starting from the WDW equation, this shows that one recuperates the notion of a Schrödingerian evolution in terms of the (coordinate-invariant) time $t_{\infty}$ when one imposes that the 3 -geometries are asymptotically flat. Note also that this equation is the quantum version of eq. (20), thereby justifying the classical analysis of that Section.

Similarly, in the presence of an inner boundary, when working at fixed opening time $\Theta$, the Wheeler-DeWitt equation is supplemented by another remarkable equation [12]:

$$
i \partial_{\Theta}|\Psi\rangle=-\frac{\hat{A}}{8 \pi}|\Psi\rangle
$$

Notice the different signs of $\hat{A}$ and $\hat{M}$ in these two equations. This is because $\hat{A}$ comes from an inner boundary and $\hat{M}$ from an outer one. This flip of sign can already be seen at the classical level by comparing eqs. (19) and (21). Indeed, eq. (30) corresponds to the quantized version of the Hamilton-Jacobi equation $\partial_{\Theta} S=A$ which follows from eq. (22). In eq. (30), the operator $\hat{A}$ is defined from the behavior of the 3 -geometries as one reaches the horizon. When the local WDW constraints are satisfied, $\hat{A}$ parametrically depends on the matter and gravitational configurations from the horizon till $r=\infty$. (A simple example of this dependence is provided by the solutions (24) of equation (30) where the eigenvalue $A$ depends on the state of the detector.)

In our derivation, we first assume that, in the absence of interactions governed by $\gamma$, the energy of the radiation field (whether or not it is in an excited state) does not influence the geometry. This amounts to assume that the wave functions of the initial and final free states factorize:

$$
\begin{aligned}
\left|\Psi_{i n}(\Theta)\right\rangle & =\left|\Psi_{B H+g}(\Theta)\right\rangle \otimes\left|0_{U}(\Theta)\right\rangle \\
\left|\Psi_{\text {fin }}(\Theta)\right\rangle & =\left|\Psi_{B H+e}(\Theta)\right\rangle \otimes\left|\Psi_{\Phi_{\omega}}(\Theta)\right\rangle
\end{aligned}
$$

This factorization is equivalent to postulate in a path integral formulation that the total action splits as a sum: $S_{B H+\operatorname{det}+\Phi}=S_{B H+d e t}+S_{\Phi}$ wherein the latter is evaluated 
in the background defined by $S_{B H+d e t}$. As shown in [34, this approximation means that the recoil of gravity due to the energy of the radiation field has been taken to account to first order only. The corrections to this linear approximation are governed by gravitational interactions of the form $\iint T G T$ where $T$ is the energy momentum tensor of the radiation field and $G$ the Green function of the background degrees of freedom 35. These interactions contain three parts. The first describes the gravitational interactions of the photon and the detector. Neglecting these is probably legitimate if the detector is far from the black hole. The second part concerns the gravitational self interactions of the photon. These will be studied in the context of the shell model of KKW in the next section and will be shown to confirm eq. (25). The third part describes the interactions among the quanta present in Unruh vacuum. In a dilute gas approximation these are neglected.

In brief, under the assumption of factorizable wave functions, the time dependence of the black hole + detector waves are given by eq. (24) in virtue of eq. (30). And eq. (25) follows from usual Unruh boundary condition. This last assumption also means that we postulate that the near horizon fluctuations have no significant effect on Unruh vacuum. One might indeed fear that these fluctuations 36, 37] would destroy its characterization. We are tempted to believe however that this is not the case. First the recent body of works initiated by Unruh on acoustic black holes 38, 39 pleads in favor of this belief. In these works indeed, it was shown that there is an adiabatic decoupling between the low energy physics governed by the surface gravity and the high energy regime. This explains why modifications of the dispersion relation at high frequencies affect neither the low energy properties of Hawking radiation nor the characterization of Unruh vacuum. That this also applies to the case of near horizon fluctuations is the subject of current work 40.

The second hypothesis concerns the transition amplitudes. To obtain them, one should incorporate the interaction Hamiltonian, eq. (17), in the Wheeler-DeWitt constraint, represented here by eqs. (28) and (30). To first order in $\gamma$, the modified propagation is expressed in terms of matrix elements of this Hamiltonian sandwiched by the free wave functions, as usual for first order in the Born series. This is also true when working with the solutions of the Wheeler-DeWitt equation[2]. Thus to first order in $\gamma$ and up to an overall factor, the transition amplitude is given by

$$
\begin{aligned}
\mathcal{B}_{g \rightarrow e+\omega} & =-i \gamma \int d \Theta\left\langle\Psi_{B H+e}\left|\left\langle\Psi_{\Phi_{\omega}}|[\hat{\Phi}(R, \Theta)|e\rangle\langle d|]| \Psi_{B H+g}\right\rangle\right| 0_{U}\right\rangle \\
& \simeq-i \gamma \int d \Theta e^{-i\left(S_{B H+e}-S_{B H+g}\right)} \times e^{-i S_{\omega}} .
\end{aligned}
$$

In the second line, we have written the phase factors in terms of the action of the black hole + detector system and that of the radiation field. This is to emphasize that only differences of actions appear in the integrand. Indeed, in the first factor, the action of the black hole alone cancels and one is left with the difference due to the change in

\footnotetext{
${ }^{2}$ In fact the derivation of eq. (32) closely follows that of transition amplitudes in quantum cosmology as performed in [11]. In both cases the wave functions appearing in matrix elements are WKB solutions of the Wheeler-DeWitt equation which govern free propagation. Another useful analogy is provided by the Unruh effect 23]. In that case, the given trajectory (which plays the role of the classical geometry in black hole physics) is replaced by WKB waves in order to take into account recoil effects 42.
} 
the detector's state. Similarly, in the second factor, $S_{\omega}$ is the change in action of the radiation field due to the insertion of the field operator at $R, \Theta$. Using the stationary character of the states for the first factor and Unruh boundary condition for the second lead to eq. (26) and hence to our central result eq. (27).

To obtain further insight about eq. (32), it is interesting to show how it gives back the conventional amplitude $B_{g \rightarrow e+\omega}$ obtained in a given background, see eq. (8). To recover this expression, it suffices to evaluate the difference in actions appearing in eq. (32) to first order in the energy change:

$$
\begin{aligned}
S_{\text {fin }}^{B H+\text { det. }+\Phi}(\Theta)-S_{\text {in }}^{B H+\text { det. }+\Phi}(\Theta) & =\omega \partial_{\omega} S_{\omega}(\Theta)+\left(E_{e}-E_{g}\right) \partial_{E_{\text {det. }}} S_{B H+\text { det. }}(\Theta) \\
& =\omega U_{K}(\Theta)+\left(E_{e}-E_{g}\right) t(\Theta) .
\end{aligned}
$$

In deriving this, we have first used the splitting of the total action discussed above and then Hamilton-Jacobi equations. Indeed, $U_{K}$ is conjugate to the frequency $\omega$ and $t$ to $E_{\text {detector }}$. It should be stressed that this recovery of the background field phases in the limit of small energy differences is a generic feature [42, 34, 19]: Whenever one takes into account a neglected heavy degree of freedom (here gravity), describes it by WKB waves and expands the resulting expressions for light transitions to first order in the light change, one recovers the usual background field expressions in which the heavy variable is treated classically.

Moreover this first order expansion "commutes" with the integration over $\Theta$. Thus it could equally be carried out after having performed the $\Theta$ integration. In this second form, one is expanding the exponential governing transition rates, eq. (27), to first order in $E_{e}-E_{g}$. In this one recovers the first law of black hole mechanics. This shows that the first law is nothing but the Hamilton-Jacobi equation applied to the Euclidean sector in the absence of conical singularity:

$$
\partial_{E_{\text {det. }}} S_{B H+\text { det. }}^{\text {Euclid. }}=\left.\partial_{E_{\text {det. }}}(A / 4)\right|_{M_{A D M}}=-2 \pi / \kappa .
$$

In view of the generic character of these features, it is clear that our analysis also applies to charged or rotating holes (see however the remark made after eq. (5)) and to cosmological or acceleration horizons. Indeed all that is required is the evaluation of the phase factors entering eq. (32). In this expression, the second factor $e^{i S_{\omega}}$ presents no difficulty: it always encodes vacuum conditions as one crosses the future horizon. The first factor is more delicate since it requires to solve Einstein's equations driven by the energy of the detector. However in the case of static Rindler like situations, the on shell action gets its contribution only from the surface term at the horizon. Moreover, since only differences appear in transition amplitudes, eq. (32) also applies to the accelerated cases. Indeed even though the area of acceleration horizon might be infinite, the change in the horizon geometry due to a finite change in the matter energy distribution is finite and well defined, see [10 for an explicit computation. In particular, it is local in the transverse directions $x_{\perp}^{i}$ when the change in the matter energy distribution is localized. This leads to finite and well defined changes in on-shell actions for the gravity-detector system which furthermore gives back the conventional background field result when linearized in $\Delta E_{\text {detect. }}$. 


\section{The KKW model}

In the previous calculation the change in area was due to the change of the detector state. However the existence of a detector is not intrinsic to black hole radiation: the detector was only used to reveal the existence of the quanta of the radiation field. Therefore we seek for an intrinsic derivation of black hole radiance in which the change in area is due to the emission process itself. In this description, the change in area plays the role of the energy of the emitted quantum in Hawking's derivation. To this end, we must introduce a model for the emitted quanta which takes into account the deformation of the gravitational background. The simplest model is that of KKW[6, 7, 8] in which one makes the hypothesis that in the semi-classical limit and at high frequency the particles are described by self gravitating spherically symmetric light-like shells.

The starting point of the KKW model is the situation analyzed in [43]. It describes the entire spherically symmetric space time, solution of Einstein's equations, which results from the propagation of a light like shall. By Birkoff's theorem, both outside and inside the shell the geometry is Schwarzschild. As in section to we shall take the outside mass $M_{A D M}$ to be fixed whereas inside the residual mass $M(\lambda)=M_{A D M}-\lambda$ depends on $\lambda$, the energy of the emitted shell measured at $r=\infty$. In both geometries the shell follows an outgoing light like geodesic, see 43. In what follows we shall use only the inner metric to describe the trajectory, the action and the wave function of the shell. This choice will be justified after having presented the results.

Inside the shell, in Eddington-Finkelstein coordinates the metric is

$$
d s^{2}=-\left(1-\frac{2 M(\lambda)}{r}\right) d v^{2}+2 d v d r+r^{2} d \Omega^{2}
$$

and the trajectory of the shell satisfies

$$
\begin{aligned}
d v & =2 \frac{d r}{1-2 M(\lambda) / r_{s h}}, \\
v\left(r_{s h}\right) & =2 r_{s h}+4 M(\lambda) \ln \left(r_{s h}-2 M(\lambda)\right) .
\end{aligned}
$$

As in the previous section it is a appropriate to introduce a dimensionless time parameter defined near the horizon. The light like version of $\Theta$ is

$$
V=\kappa(\lambda) v=\Theta+\kappa(\lambda) r+\frac{1}{2} \ln \left(r-r_{H}(\lambda)\right)
$$

where $\kappa(\lambda)=\frac{1}{4 M(\lambda)}$ is the surface gravity and $r_{H}(\lambda)=2 M(\lambda)$ is the final radius of the horizon. We shall also use as energy variable the area of the horizon $A=\pi r_{H}^{2}(\lambda)$ rather then $\lambda$ because $A / 8 \pi$ is the conjugate to $\Theta$ and to $V$. In terms of these new variables the trajectory is

$$
V\left(r_{s h} ; A\right)=\ln \left(r-r_{H}(A)\right)+2 \kappa(A) r \simeq \ln \left(r-r_{H}(A)\right)+O\left(r-r_{H}(A)\right) \quad .
$$

Close to the horizon the log dominates and the trajectory is expressed only in terms of quantities locally defined. Therefore this expression characterizes radial trajectories 
near all event horizons. As an illustration of this universality one easily verifies that for a charged non extremal black hole, the trajectory near the horizon also takes the form $V \simeq \ln \left(r-r_{H}\right)$ wherein the surface gravity does not appear. As in the former Section, we make the hypothesis that the near horizon fluctuations do not alter the analytical behavior of this trajectory.

In order to obtain the modes characterizing the quantum propagation of this shell we need its action. To obtain the action one could start from scratch, that is from the Einstein-Hilbert plus matter action and extremise it. This is the path followed in [6, 7]. However, since we know the trajectories we can use a short cut to obtain the Hamilton-Jacobi action, see also [8].

Since the inside geometry is static, $A$ is a constant of motion. Therefore the action can be written as

$$
S(r, V ; A)=\frac{A V}{8 \pi}+f(r, A)
$$

where $f$ is the Maupertuis action, that is $\partial_{r} f=p$ is the momentum of the shell. The classical trajectory follows from stationarity of $S$ with respect to $A, \partial_{A} S=0$. In the present case it implies that $\partial_{A} f=-V(r ; A) / 8 \pi$ where $V$ is given by eq. (38). After integration from $A$ to $A_{0}$, one obtains

$$
S(r, V ; A)=\frac{\left(A-A_{0}\right) V}{8 \pi}+\int_{A}^{A_{0}} \frac{d \tilde{A}}{8 \pi}\left(\ln \left(r-r_{H}(\tilde{A})\right)+\kappa(\tilde{A}) r\right)-g\left(r_{0}\right)
$$

$A_{0}$ is the area of the horizon in the absence of shell, equal to $A_{0}=4 \pi M_{A D M}^{2}$. With this choice, in the absence of the shell the action vanishes. This guarantees that to first order in the shell energy $\lambda$ one identically recovers the action of a massless particle in the background geometry (at this order it can be taken to be either the inner or outer geometry). Notice also that $A_{0}$ is larger then $A$ since the area of the horizon decreases when a particle of positive energy is emitted.

We have also added an $r$ and $V$ independent integration constant

$$
g\left(r_{0}\right)=\int_{A}^{A_{0}} \frac{d \tilde{A}}{8 \pi}\left(\ln \left(r_{0}-r_{H}(\tilde{A})\right)+\kappa(\tilde{A}) r_{0}\right)
$$

At fixed $A$ this function enables the initial momentum $p_{0}$ to be fixed arbitrarily and determine implicitly the initial radius $r_{0}$ or conversely to fix $r_{0}$ and determine implicitly $p_{0}$. Technically this follows from $\partial_{r_{0}} S=-p_{0}\left(A, r_{0}\right)$.

The action eq. (40) is well defined on either side of the smeared horizon, that is for both $r$ and $r_{0}$ greater than $r_{H}\left(A_{0}\right)$ or both less than $r_{H}(A)$. In each region, we can use it to define (in the WKB approximation) the wave function of self gravitating shells. For $r$ and $r_{0}$ greater than $r_{H}\left(A_{0}\right)$, one has

$$
\varphi_{\Delta A,+}=e^{i S(V, r ; \Delta A)} \theta\left(r-r_{H}\left(A_{0}\right)\right)
$$

This describes the classically allowed propagation of a shell outside the horizon. Similarly we can define a wave function

$$
\varphi_{\Delta A,-}^{*}=e^{i S(V, r ; \Delta A)} \theta\left(r_{H}(A)-r\right)
$$


living only in the region $r, r_{0}<r_{H}(A)$. These definitions are in strict analogy with the wave functions $\varphi_{\lambda \pm}$ defined in eq. (11) and reduce to them in the limit $\lambda \rightarrow 0$. In particular the complex conjugation in eq. (43) arises because behind the horizon $\Theta$ time runs backwards. Notice that we did not write the prefactors of these waves. To conform ourselves to second quantized rules, we should have introduced prefactors such that the Wronskian be unity. We shall not pursue this since the prefactors play no role in what follows, i.e. the determination of the pair creation probability.

Between $r_{H}\left(A_{0}\right)$ and $r_{H}(A)$ one needs a prescription to define the logarithm. As explained at the end of section 2, the definition of the action can be used to encode the Unruh boundary condition. The analytical specification imposes that the $\phi_{\Delta A,+}$ mode, the analog of $\phi_{\lambda,+}$ of eq. (13),

$$
\phi_{\Delta A,+}=e^{i S_{U}(V, r ; \Delta A)}
$$

be analytical and bounded in the upper half of the complex $r$ plane at fixed $V$. This leads to the globally defined action

$$
\begin{gathered}
S_{U}\left(r, V ; A, r_{0}>r_{H}\left(A_{0}\right)\right)=\frac{\left(A-A_{0}\right) V}{8 \pi} \\
+\int_{A}^{A_{0}} \frac{d \tilde{A}}{8 \pi}\left[\ln \left|r-r_{H}(\tilde{A})\right|+i \pi \theta\left(r_{H}(\tilde{A})-r\right)+O\left(r-r_{H}(\tilde{A})\right)\right]-g\left(r_{0}\right) .
\end{gathered}
$$

Thus as $r$ goes from $r>r_{H}\left(A_{0}\right)$ to $r<r_{H}(A)$ the action acquires an imaginary part equal to

$$
\operatorname{Im} S_{U}=\frac{A_{0}-A}{8}
$$

This simple result is due to the fact that the log in eq. (40) comes with an $\tilde{A}$ independent weight. As emphasized after eq. (38) the origin of this independence follows from the universal form of light like outgoing trajectories near a future horizon: $V=\ln \left(r-r_{H}(A)\right.$ ). Therefore eq. (46) universally follows from this behavior and from Unruh's prescription for the analytical behavior of the modes. This is the essential kinematical result of this section.

Using this result, we can write $\phi_{\Delta A,+}$ as the linear combination

$$
\phi_{\Delta A,+}=\varphi_{\Delta A,+}+e^{-\Delta A / 8} \varphi_{\Delta A,-}^{*} .
$$

wherein only the relative weight of $\varphi_{\Delta A,+}$ and $\varphi_{\Delta A,-}^{*}$ has meaning, c.f. the above discussion about the normalization of the Wronskian. In section 2, the technique of Bogoljubov transformation enabled us to identify the square of the ratio of the weights of $\varphi_{+}$and $\varphi_{-}^{*}$ with the probability to emit a pair, see eq. (15). In the presence of backreaction, the same relation still holds for rare and energetic events. Thus we obtain

$$
P_{\Delta A}=e^{-2 \operatorname{Im} S_{U}}=e^{-\Delta A / 4}
$$

in place of eq. (15) and in agreement with eq. (27) and [8]. This result can probably be generalized to rotating holes and to cosmological or acceleration horizons. But this 
will require that one refines the procedure so as to take into account the transversal directions. We hope to return to this problem.

It should be stressed that the justification of the identification of $e^{-2 \operatorname{Im} S_{U}}$ as the transition probability is more delicate in the presence of backreaction than in the free field theory. This point is discussed below.

\section{Discussions}

To prepare the discussion, it is appropriate to compare our treatment with the original derivation of KKW [6, 7, 8]. A first difference with the calculation of $\mathrm{KKW}$ is that we use $A, V$ instead of $\lambda, t$ as energy and time variables. The passage from one to the other is straightforwardly implemented by using the $\lambda$-dependent Jacobian $d A / d \lambda$ in the action. The advantage of using the $A, V$ variables from the start is that it is then manifest that the result for the probability of emission $P=e^{-\Delta A / 4}$ is no accident, but follows from the universal form of classical trajectories near a horizon.

A second difference is that KKW work with fixed black hole mass $M$ and with a varying $\mathrm{ADM}$ mass $M+\lambda$. In an empty geometry, this can be shown to be mathematically equivalent to working as we do with a varying black hole area and fixed ADM mass. However as discussed in section 3 the second description reflects better the physics of the emission process wherein the black hole loses energy to the radiation while the mass at infinity stays constant. Then, when working at fixed ADM mass, the time at infinity cannot be used to parameterize the propagation of the shell, see [18] for a general proof of this super-selection rule. Thus, as in Section 4, it is through the $\Theta$ (or $V$ ) dependence that one recovers the notion of evolution. For non empty geometries, we conjecture that it will be mandatory to work with a varying horizon area, i.e. to use the variables $A, V$ to parameterize the process.

Thirdly we have implemented the Unruh boundary condition on the area eigenstates $\phi_{\Delta A, \pm}$ through the analytical behavior in $r$ as it goes from one side of the horizon to the other. In [8] instead, the Unruh boundary condition is implemented by fixing the initial momentum $p_{0}=-\partial_{r_{0}} S$ at $V=0$. These two procedures are equivalent. Indeed, in both cases, the definition of $\ln \left(r-r_{H}\right)$ in the action is obtained by imposing positive frequency in $i \partial_{r}$ across the horizon.

The boundary condition of fixed large positive momentum $p_{0} \gg \lambda$ also shows the relation with the approach of section 4 . Indeed the action of a self gravitating shell with initial constant momentum $p_{0}$ can be shown to be

$$
S(r, V)_{p_{0}}=\left[p_{0} r+h\left(p_{0}\right)\right] e^{-V} .
$$

The assumption of KKW that in the presence of backreaction modes have the form $\phi=e^{i S_{p_{0}}}$ is therefore equivalent to the assumption made in section 4 , see eq. (25), that the wave function of particles in Unruh vacuum is proportional to $e^{i C \omega e^{-\Theta}}$ at constant $r$.

3 This is obtained by replacing the equation for the trajectory near the horizon $V=\ln \frac{r-r_{H}(A)}{r_{0}-r_{H}(A)}$ in the equation for the momentum $p=\int_{A}^{A_{0}} \frac{d \tilde{A}}{8 \pi} \frac{1}{r-r_{H}(\tilde{A})}$ to obtain $p=p_{0} e^{-V}$. Integrating the HamiltonJacobi equation $\partial_{r} S=p$, one finds $S_{p_{0}}=p_{0} r e^{-V}+F\left(p_{0}, V\right)$ where $F$ is an $r$ independent integration constant. Then imposing that on the equations of motion the action be stationary with respect to variations of $p_{0}$, one finds eq. (49) with $\partial_{p_{0}} h\left(p_{0}\right)=r_{H}\left(r_{0}, p_{0}\right)$. 
We now turn to the delicate question of identifying twice the imaginary part of the action to go from one side of the horizon to the other with the (log of the) probability to emit a pair. In addressing this question one faces a double problem. First, due to gravity, the emission process is no longer linear. Indeed when two shells of energy $\lambda$ are emitted, their action is not twice that of one particle. Therefore, probabilities for multi-particle production are no longer obtainable from those for single particle emission as they are in a linear field theory. This means that the machinery of Bogoljubov transformations no longer applies. The second problem is due to the use of WKB approximations for the wavefunctions which are given in terms of the action of a single shell. Because of this we are certainly not in a position to describe multi-particle effects, that is higher order effects in the tunneling amplitude $e^{-\operatorname{Im} S_{U}}$.

We shall now sketch how one can deduce, to leading order in $e^{-I m S_{U}}$, the probability of particle production from the properties of the wave functions, without resorting to Bogoljubov transformations. To present our method, we first return to the analysis in the absence of backreaction and consider the following matrix element

$$
\begin{aligned}
\frac{\left\langle 0_{U}\left|\int d \tilde{v} e^{i \lambda \tilde{v}} \Phi(R=+\infty, \tilde{v}) \Phi(r, v)\right| 0_{U}\right\rangle}{\alpha_{\lambda}^{2}} & =\frac{\left\langle 0_{U}\left|a_{o u t, \lambda+} \Phi(r, v)\right| 0_{U}\right\rangle}{\alpha_{\lambda}^{2}} \\
& =\frac{\phi_{\lambda+}^{*}(r, v)}{\alpha_{\lambda}}=\varphi_{\lambda+}^{*}(r, v)+\frac{\beta_{\lambda}}{\alpha_{\lambda}} \varphi_{\lambda-}(r, v)
\end{aligned}
$$

When $r>2 M$, it has a simple interpretation. It is the amplitude for an out-particle created at $(r, v)$ to be found at $\mathcal{I}^{+}$with energy $\lambda$. This is a classical process governed by the action $\int_{r}^{+\infty} d r^{\prime} p_{\lambda}\left(r^{\prime}\right)$ where $p_{\lambda}=2 \lambda /(1-2 M / r)$ is the classical momentum of the particle. The normalization has been chosen so as to describe one out-particle, i.e. to have a unit current (Wronskian) for $r>2 M$. In a linear field theory this normalization can be calculated exactly and is given by the factors of $\alpha_{\lambda}$, see eq. (13). It is thus proportional to $1+O\left(e^{-2 \pi \lambda / \kappa}\right)$. For self-gravitating shells, this normalization might be different but will remain proportional to $1+O\left(e^{-2 \operatorname{Im} S_{U}}\right)$.

When $r<2 M$, this matrix element has also a simple interpretation because $\Phi(r, v)$ and $a_{\text {out }, \lambda+}$ commute. It defines the amplitude for a pair to be emitted by the black hole. Indeed, it defines the transition amplitude from $\left|0_{U}\right\rangle$ to the state $\Phi(r, v) a_{\text {out }, \lambda+}^{\dagger}\left|0_{U}\right\rangle / \alpha_{\lambda}^{2}$. One member of the pair is outside the horizon and has energy $\lambda$, the other is behind the horizon at $(r, v)$. ¿From this we deduce that the probability $P_{\lambda}$ to create a pair is given by the current $\beta_{\lambda}^{2} / \alpha_{\lambda}^{2}$ carried by $\phi_{\lambda+}^{*} / \alpha_{\lambda}$ for $r<2 M$.

This expression for $P_{\lambda}$ can in turn be used to show how $P_{\lambda}$ can be expressed in terms of the classical action. Recall that the prescription for defining the Unruh mode $\phi_{\lambda+}$ for $r<2 M$ is such that its phase, i.e. the action $\int p_{\lambda} d r$, is obtained by analytically continuing $r-2 M$ in the upper half complex plane at fixed $v$. This fixes the ratio of the amplitudes $\phi_{\lambda+}$ on each side of the horizon. Thus the probability $P_{\lambda}$ is given by

$$
P_{\lambda}=\frac{\left|\phi_{\lambda,+}(r>2 M, v)\right|^{2}}{\left|\phi_{\lambda,+}(r<2 M, v)\right|^{2}}=e^{-2 \operatorname{Im} \int_{r<2 M}^{r>2 M} d r^{\prime} p_{\lambda}\left(r^{\prime}\right)}=e^{-2 \pi \lambda / \kappa}
$$

Note that both the normalization $\alpha_{\lambda}$ and the relativistic prefactors $(4 \pi \lambda)^{-1 / 2}$ cancel in 
this ratio. This follows from the fact that $P_{\lambda}$ is given by the ratio of the currents carried by $\phi_{\lambda,+}$ on each side of the future horizon.

Upon taking into account the gravitational backreaction, the above argument still applies if two conditions are met. First the WKB approximation of the wave functions must be valid. This guarantees that we can use the classical action to evaluate the relative amplitude of the Unruh modes on each side of the horizon. Secondly, the wave function of the total system, black hole + radiation field, must be (approximatively) factorisablef into the wave function of the shell times the rest. Both require that the energy of the shell be large, i.e. $\lambda / \kappa \gg 1$.

In quantum mechanics such a factorization is a good approximation whenever there is a hierarchy in the degrees of freedom: very heavy degrees of freedom that can be treated in the WKB approximation (the nuclear degrees of freedom in the case of a Rydberg electron orbiting an atom or molecule, the radius of the universe in quantum cosmology, the black hole in the present case); moderately energetic degrees of freedom that can also be treated in the WKB approximation (the Rydberg electron, heavy matter in cosmology, rare energetic particles emitted by the black hole); and degrees of freedom that must be treated quantum mechanically (the inner electrons, the other matter degrees of freedom in cosmology, less energetic particles emitted by the black hole). The very heavy and the moderately heavy degrees of freedom propagate semi-classically in the mean potential due to the other degrees of freedom (for a detailed treatment of the Born-Oppenheimer approximation in a dynamical context, see 44 for atomic physics and 450 for cosmology). Moreover, the probabilities of the rare quantum transitions of the moderately energetic degrees of freedom can be obtained from the sole properties of their wave function. The reason is that the wave functions of both the heavier and the lighter degrees cancel out from the bra-kets which represent transition amplitudes. An illustration of such cancellations is given in eq. (32) where only the difference in actions due to the process itself enter in the transition amplitude.

In brief, when both conditions are met, the former analysis performed in the linear theory applies. This guarantees that to first order in $e^{-I m S_{U}}$, eq. (48) is correct. This is also what has been adopted in [6, 7, 8].

\footnotetext{
${ }^{4}$ When the shell is very close to the horizon, this factorization probably breaks down because of its momentum $\partial_{r} S\left(=p_{\lambda}(r)\right.$ in a given background) is arbitrary large (trans-Planckian) since it diverges. In the presence of backreaction, the new momentum $\partial_{r} S_{U}$ diverges logarithmicly as $r \rightarrow r_{H}(A)$, see eq. (45). However, once $\partial_{r} S_{U}$ is much smaller than the Planck mass, the factorization should apply. The crucial point is the existence of an intermediate region in which the log is dominating the action $S_{U}$, this requires $\partial_{r} S_{U} \gg \lambda$, and in which the factorization applies, this requires $\partial_{r} S_{U} \ll M_{\text {Planck. }}$. In this region, the usual analytical characterization of Unruh vacuum still applies 47 and this is sufficient to obtain eq. (48). It is interesting to notice that the same logarithmic behavior (in an intermediate region) also explains the absence of modifications to Hawking radiation when one mutilates the dispersion relation 38, 39]
} 


\section{Conclusion}

Let us further discuss the hypothesis we made. First we supposed that, in the presence of backreaction, physics in the neighborhood of a black hole is described by the total action $S_{\text {gravity+matter }}$. Second we supposed that the wave function of the total system factorizes into a piece describing the emitted particle and the rest describing the black hole and the other matter degrees of freedom. Finally we supposed that the wave functions governing heavy degrees of freedom could be approximated by a WKB form.

The first hypothesis may seem completely evident, but one must recall that there are scenarios, such as the brick wall model of 't Hooft, in which it is not necessarily true. Furthermore a recent critique of the KKW model [46] does not recover eq. (1) because its matter model does not derive from an action principle. The validity of the last two hypothesis was discussed at the end of the preceding section and in [7].

Upon making these hypothesis one finds that the probability of the black hole of emitting a particle of energy $\lambda$ is given by

$$
P_{M \rightarrow M-\lambda}=N(\lambda, M) e^{-\Delta A(\lambda, M) / 4} .
$$

We have emphasized the universal validity of this result by giving two different derivations. Its importance lies in two facts. First it provides statistical foundations to black hole thermodynamics since it applies to every quantum emission and since it is governed by the induced quantum change (i.e. the recoil) of the horizon area. But, because we have not identified the microcanonical states, and because we work in a WKB approximation, it is only valid in a mesoscopic sense discussed below. Secondly since the derivation of eq. (52) also applies to any quantum emission arising in the presence of an event horizon, this statistical interpretation is also valid for all event horizons.

To appreciate the first fact, let us recall that the first law 11] of black hole thermodynamics is a purely classical result which is obtained by comparing two slightly different solutions of Eintein's equations. On the other hand Hawking's derivation of black hole radiation is obtained by quantizing a matter field in a given geometry. Thus, there is no a priori reason why these two results obtained in so different settings should be related or even compatible. It turns out however that they are consistent with each other and this is the basis for the successful thermodynamics of black holes.

The origin of this complementarity can be easily understood from the "improved" derivation of Hawking radiation presented here. Since energy conservation is implemented from the start, one takes into account the change of the black hole geometry due to the emission itself and one finds that the emission probability is governed by a difference of matter+gravity actions $S_{m+g}($ final $)-S_{m+g}($ initial $)=\Delta A / 4$. From this macrocanonical result one recovers the canonical distribution found by Hawking by expanding this difference to first order in the energy change $\lambda$. In the same way the first law is also obtained in [12] by this expansion. It is therefore a mathematical necessity that they be consistent.

The other important aspect of eq. (52) is that it indicates that black hole thermodynamics is not only valid in the mean (in the sense of a large ensemble of processes) 
but that it applies to every quantum mechanical process. Recall that quantum mechanics predicts that ratio of the decay rate to the absorption rate of the same quantum is proportional to the ratio of the number of final to initial micro-states (at least when the interaction Hamiltonian can be treated as a constant). When this is the case, eq. (52) leads to the identification

$$
\Delta A / 4=\text { the change of the ln of the nbr. of horizon states . }
$$

Moreover, since eq. (52) also applies to quantum emissions near all event horizon, eq. (59) also applies to all event horizons. The universality of this identification invites three comments.

Firstly, it leads to the notion of entropy density [48, 32] per unit horizon area. Indeed, when considering local creation processes rather than global ones described by $s$-waves, the change in area is local [10] in the transverse directions $x_{\perp}^{i}$. This means that a finite and localized set of horizon states are affected by the process.

Secondly, since eq. (53) applies to acceleration horizons, i.e. to near vacuum configurations having entropy measured by inertial means that can be very small, one is lead to conclude that $A / 4$ determines the number of states available to the (accelerated) system in contact with the event horizon, i.e. with its heat. If this is the case, it implies that the black hole area should be viewed as a measure of the entropy accessible to the external world [20, 22]. How to define its 'actual' entropy and whether this latter will still be given by $A / 4$, as is found for extremal black holes in string theory, [1] are important questions.

Thirdly, it should be stressed that the identification expressed by eq. (53) says nothing about the microscopic nature of the degrees of freedom which constitute the states of event horizons entering in the transition amplitudes we computed. All we can say is that when the three hypothesis we made are valid, the number of horizon states must obey eq. (53). Therefore, eq. (53) must be conceived as a universal mesoscopic [50, 21] result, somehow intermediate between the classical laws of thermodynamics and the ultimate theory of quantum gravity.

Acnowledgements The authors would like to thank Roberto Balbinot and Ted Jacobson for enjoyable discussions. They also thank the referee and R. Brout for their criticisms which helped to clarify the manuscript. S.M. is a "chercheur qualifié du FNRS". He would like to thank Utrecht university where part of this work was carried out.

\section{References}

\footnotetext{
${ }^{5}$ As an additional point, recall that Hawking radiation enlarged the domain of validity of the generalized second law to processes where the black hole temperature can be treated as a constant 49. We conjecture that eqs. (52) and (53) can be used to enlarge its domain of validity to situations in which the canonical approximation breaks down, both for black holes and other horizons.
} 
[1] J. M. Maldacena, Black Holes in String Theory, hep-th/9607235; A. W. Peet, Class. Quant. Grav. 15 (1998) 3291

[2] S. W. Hawking, Comm. Math. Phys. 43 (1975) 199

[3] J. M. Bardeen, Phys. Rev. Lett. 46 (1981) 382

[4] R. Parentani and T. Piran, Phys. Rev. Lett. 73 (1994) 2805

[5] S. Massar, Phys. Rev. D 52 (1995) 5861

[6] P. Kraus and F. Wilczek, Nucl. Phys. B 433 (1995) 403

[7] P. Kraus and F. Wilczek, Nucl. Phys. B 437 (1995) 231

[8] E. Keski-Vakkuri and P. Kraus, Nucl. Phys. B 491 (1997) 249

[9] S. Massar and R. Parentani, Phys. Rev. Lett. 78 (1997) 3810

[10] S. W. Hawking, G.T. Horowitz and S. Ross, Phys. Rev. D51 (1995) 4302

[11] R. M. Wald, Quantum Field Theory in Curved Spacetime and Black Hole Thermodynamics, Univ. of Chicago Press, 1994.

[12] S. Carlip and C. Teitelboim, Class. Quant. Grav. 12 (1995) 1699

[13] C. Teitelboim, Phys. Rev. D 51 (1995) 431

[14] C. Teitelboim, Phys. Rev. D 53 (1996) 2870

[15] S. Carlip and C. Teitelboim, Phys. Rev. D 51 (1995) 622

[16] M. Banados, C. Teitelboim and J. Zanelli, Phys. Rev. Lett. 72 (1994) 957

[17] G. W. Gibbons and S. W. Hawking, Phys. Rev. D15 (1977) 2752

[18] D. P. Page and W. K. Wooters, Phys. Rev. D27 (1983) 2885

[19] Cl. Gabriel, Ph. Spindel, S. Massar and R. Parentani, Phys. Rev. D57 (1998) 6496

[20] see the conclusions in [4]

[21] R. D. Sorkin, gr-qc/9705006, in Black Holes and Relativistic Stars, edited by R.M. Wald (University of Chicago Press, 1998)

[22] T. Jacobson, Phys. Rev. Lett. 75 (1995) 1260, see also gr-qc/9908031

[23] W.G. Unruh, Phys. Rev. D 14 (1976) 870

[24] G.W. Gibbons, Commun. Math. Phys. 44 (1975) 245 
[25] N.D. Birrel and P.C.W. Davies, Quantum Fields in Curved Space, Cambridge University Press, 1982.

[26] R. Parentani and R. Brout, Int. J. Mod. Phys. D 1 (1992) 169

[27] R. Brout, S. Massar, R. Parentani and Ph. Spindel, Phys. Rep. 260 (1995) 329

[28] T. Damour and R. Ruffini, Phys. Rev. D 14 (1976) 332

[29] T. Regge and C. Teitelboim, Ann. of Phys. 88 (1974) 286

[30] K. V. Kuchar, Phys. Rev. D 50 (1994) 3961

[31] M. Parikh, F. Wilczek, Phys. Rev. D 58 (1998) 064011

[32] S. Carlip, Class. Quant. Grav. 16 (1999) 3327

[33] A. Ashtekar, A. Corichi and K. Krasnov, gr-qc/9905089

[34] R. Parentani, gr-qc/9803045, to appear in Class. Quantum Grav.

[35] A. O. Barvinski and C. Kiefer, Nucl. Phys. B525 (1998) 509

[36] R. D. Sorkin, gr-qc/9508002, in Proceedings of the Conference on Heat Kernel Techniques and Quantum Gravity, edited by S.A. Fulling, University of Texas Press (1995)

[37] A. Casher, F. Englert, N. Itzhaki, S. Massar, R. Parentani, Nucl. Phys. B 484 (1997) 419

[38] W. G. Unruh, Phys. Rev. D 51 (1995) 2827

[39] R. Brout, S. Massar, R. Parentani, P. Spindel, Phys. Rev. D 52 (1995) 4559

[40] C. Barrabès, V. Frolov and R. Parentani, in preparation

[41] R. Parentani, Nucl. Phys. B. 492 (1997) 501,

[42] R. Parentani, Nucl. Phys. B 454 (1995) 227, see also gr-qc/9710059

[43] W. Israel, Nuovo Cimento 44 b, 1 (1966) and Phys. Rev. 153, 1388 (1967)

[44] J. B. Delos, W. R. Thorson and S. K. Knudson, Phys. Rev. A 6 (1972) 709

[45] S. Massar and R. Parentani, Nucl. Phys. B 513 (1998) 375

[46] H. Zaidi and J. Gegenberg, Phys. Rev. D 57 (1998) 1112

[47] T. Jacobson, Phys. Rev. D44 (1991) 1731, D48 (1993) 728

[48] C. Teitelboim, hep-th/9405199 
[49] W. Unruh and W. G. Wald, Phys. Rev. D 25 (1982) 942; Phys. Rev. D 27 (1983) 2271.

[50] N. G. van Kampen, Stochastic processes in Physics and Chemistry, North Holland, Elsevier (1981) 